\title{
Cortical dysplasia-focal epilepsy syndrome
}

INSERM

\section{Source}

INSERM. (1999). Orphanet: an online rare disease and orphan drug data base. Cortical dysplasia-focal epilepsy syndrome. ORPHA:163681

Cortical dysplasia-focal epilepsy syndrome is a rare genetic epilepsy characterized by relatively large head circumference or macrocephaly, diminished or absent deep-tendon reflexes and mild gross motor delay in infancy, followed by intractable focal seizures with language regression, behavioral abnormalities (hyperactivity, attention deficit, aggressive/autoaggressive behavior, autistic features) and intellectual disability later in life. 